\title{
LINEAR SUPERPOSITION OF SMOOTH FUNCTIONS
}

\author{
ROBERT KAUFMAN ${ }^{1}$
}

ABSTRACT. We give a simple proof of the impossibility of representing an arbitrary continuous function as a superposition (1), when $F_{1}, \cdots, F_{N}$ are smooth mappings of $R^{n+1}$ to $R^{n}$. The main tool is the Riemann-Lebesgue lemma.

Let $V$ be an open subset of Euclidean space $R^{n+1}$, and $F_{1}, \ldots, F_{N}$ continuously differentiable mappings of $V$ into $R^{n}$. Each $N$-tuple of bounded continuous functions $\left(g_{1}, \cdots, g_{N}\right)$ defined on $R^{n}$ determines a superposition

$$
T\left(g_{1}, \cdots, g_{N}\right)=\sum_{1}^{N} g_{k} \circ F_{k} .
$$

This is an element of the Banach space $C(V)$ of functions bounded and continuous on $V$.

Theorem. The range of the operator $T$ is of first category in $C(V)$, whence the superpositions (1) do not exhaust $C(V)$.

This theorem was proved for $n=2$ by Vitushkin and Henkin [5]; in fact they proved that the range of $T$ is not even dense in $C(V)$. For $n=$ 3 a stronger result was obtained by Fridman, but only for mappings of class $C^{2}(V)[2]$. The theorem to be proved is neither implied by the work cited, nor does it imply the strongest results known in special cases. See al so [1], [3], [4].

1. Let $x_{0}$ be a generic point for $F_{1}, \ldots, F_{N}$, that is, the rank of each Jacobian matrix $J\left(F_{k}\right)$ attains a local maximum at $x_{0}$. Such points form a dense $G_{\delta}$ in $V$. Then $J\left(F_{k}\right)$ has rank $d_{k}$ throughout a neighborhood $W$ of $x_{0}$ and (for an appropriate $W$ ) $F_{k}$ can be factored through: $R^{d_{k}}: F_{k}=$

Received by the editors October 15, 1973.

AMS (MOS) subject classifications (1970). Primary 26A72; Secondary 46E 15.

Key words and phrases. Smooth functions, Kolmogorov superposition theorem, Baire category.

${ }^{1}$ The author is an Alfred P. Sloan Fellow. 
$G_{k} \circ H_{k}$, where $H_{k}$ maps $W$ into $R^{d_{k}}$. In the superpositions (1) we can replace $F_{k}$ by $H_{k}$, provided we allow functions $g_{k}$ defined on $R^{d_{k}}$. Let $L$ be a linear functional on $R^{n+1}$ whose Jacobian matrix contains a row not spanned by $J\left(F_{k}\right)\left(x_{0}\right)$ for each individual function $F_{k}$. On a small neighborhood, each mapping $H_{k}^{*}=\left(L, H_{k}\right)$ of $W$ into $R^{d_{k}+1}$ is absolutely continuous in the following sense: there is a nonnegative function $\phi_{k}$ on $R^{d_{k}+1}$ and an identity

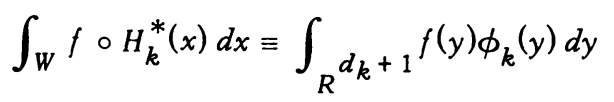

whenever $f \geq 0$ is measurable on $R^{d_{k}+1}$. Using local coordinates on $R^{n+1}$ we can obtain a refinement of the absolute continuity: let $\psi$ be continuous and have compact support in $W$; then the function $\psi_{k}$ defined by $\int f \circ H_{k}^{*}(x) \psi(x) d x$ is continuous on $R^{d_{k}+1}$ (and has compact support).

2. For computations it is convenient to use coordinates $(t, u)$ in $R^{d_{k}+1}$ : $t$ is real and $u$ is in $R^{d_{k}}$.: We apply the change of variable formula above, with $f(t, u)=e^{i \lambda t} h(u)$; for the moment $h \in L^{\infty}\left(R^{d_{k}}\right)$. This yields

$$
\int e^{i \lambda L} b \circ H_{k}(x) \psi(x) d x=\iint e^{i \lambda t} b(u) \psi_{k}(t, u) d t d u .
$$

We now suppose that $h=0$ outside $H_{k}(W)$, since this leaves $H_{k} \circ b$ unchanged. The last integral has a modulus not exceeding

$$
\|b\|_{1} \sup \left|\int e^{i \lambda t} \psi_{k}(t, u) d t\right| \equiv\|b\|_{1} \cdot M_{k}(\lambda),
$$

say. But $M_{k}(\lambda) \rightarrow 0$ as $\lambda \rightarrow+\infty$, because $\psi_{k}(t, u)$ has compact support in $R^{d_{k}+1}$-this is a simple extension of the Riemann-Lebesgue lemma. Because $\int\left|e^{i \lambda L} \psi(x)\right| d x=c^{\prime}>0$ for all $\lambda$, we have a stronger version of the main theorem:

The set $T_{1}$ of superpositions $T\left(g_{1}, \cdots, g_{N}\right)$, with $\left\|g_{k}\right\|_{1} \leq 1$ in $L^{1}\left(R^{d_{k}}\right)$, has a closure $\bar{T}_{1}$ in $L^{1}(W)$, nowhere dense in $C(\bar{W})$.

\section{REFERENCES}

1. B. L. Fridman, An improvement in the smoothness of functions in $A$. $N$. Kolmogorov's theorem on superposition, Dokl. Akad. Nauk SSSR 177 (1967), 1019 1022 = Soviet Math. Dokl. 8 (1967), 1550-1553. MR 37 \#663.

2. - A nowhere dense space of linear superpositions of functions of several variables, Izv. Akad. Nauk SSSR Ser Mat. 36 (1972), 814-846 = Math. USSR Izv. 6 (1972), 807-837. 
3. T. Hedberg, The Kolmogorov superposition theorem, Topics in Approximation Theory, Appendix II, Lecture Notes in Math., vol. 187, Springer-Verlag, Berlin and New York, 1971, pp. 267-275.

4. D. A. Sprecher, An improvement in the superposition theorem of Kolmogorov, J. Math. Anal. Appl. 38 (1972), 208-213. MR 46 \#1981.

5. A. G. Vituškin and G. M. Henkin, Linear superpositions of functions, Uspehi Mat. Nauk 22 (1967), no. 1 (133), 77-124 = Russian Math. Surveys 22 (1967), no. 1, 77-125. MR 38 \#6010.

DEP ARTMENT OF MATHEMATICS, UNIVERSITY OF ILLINOIS, URBANA, ILLINOIS 61801 